\title{
Importancia y efecto de los factores financieros y asociados a la financiación en la intensidad de la innovación de las pymes colombianas
}

\author{
Padilla-OSPina, AnA Milena \\ Universidad del Valle (Colombia) \\ Correo electrónico: ana.milena.padilla@correounivalle.edu.co \\ MEDINA-VÁSQUEZ, JAVIER ENRIQUE \\ Universidad del Valle (Colombia) \\ Correo electrónico: javier.medina@correounivalle.edu.co \\ OSPINA-HOLguíN, JAVIER HUMBERTO \\ Universidad del Valle (Colombia) \\ Correo electrónico: javier.ospina@correounivalle.edu.co
}

\begin{abstract}
RESUMEN
La financiación de la innovación se ha caracterizado por ser un campo fragmentado donde coexisten múltiples teorías, cada una enfocada en uno o en unos pocos aspectos de la financiación a la vez. El presente artículo busca identificar las variables financieras que más influyen y su efecto en la intensificación de las actividades de innovación de las pymes colombianas en un único modelo empírico comprehensivo que predice la intensidad de la innovación a partir de más de cuarenta subvariables asociadas a la financiación de la innovación. El modelo de predicción (que logró un coeficiente de determinación por fuera de la muestra sobre 0.5 o 0.6 ), según la definición utilizada, se basa en un conjunto de redes neuronales optimizadas mediante técnicas bayesianas. Los resultados de este trabajo sugieren como conclusión que para que las pymes decidan intensificar sus actividades de innovación predominan las variables capaces de limitar la innovación, como el costo de ajuste y algunos obstáculos para innovar, pero sobre todo las variables centradas en el riesgo, como el riesgo de quiebra de la empresa o del proyecto de innovación y la aversión al riesgo del gerente.
\end{abstract}

Palabras clave: financiación de la innovación, intensidad de la innovación, redes neuronales, pymes.

Clasificación JEL: O31; G32; L60; L80.

MSC2010: 68T07.

Artículo recibido el 21 de julio de 2021 y aceptado el 12 de agosto de 2021. 


\title{
Importance and effect of financial and financing- related factors on the innovation intensity of Colombian SMEs
}

\begin{abstract}
Innovation financing has been characterized as a fragmented field where multiple theories coexist, each focusing on one or a few aspects of financing at the same time. This article aims to identify the most influential financial variables and their effect on the intensification of innovation activities of Colombian SMEs, in a single comprehensive empirical model that predicts the intensity of innovation from more than forty subvariables associated with the financing of innovation. The prediction model (which achieved an out-of-sample determination coefficient of 0.5 or 0.6 ), according to the definition used, is based on a set of neural networks optimized using Bayesian techniques. The results of this work suggest, as a conclusion, that for SMEs to decide to intensify their innovation activities, variables capable of limiting innovation predominate, such as the adjustment costs and some obstacles to innovating, but especially variables focused on risk, such as the risk of bankruptcy of the company or of the innovation project and the risk aversion of the CEO.
\end{abstract}

Keywords: financing of innovation, intensity of innovation, neural networks, SMEs.

JEL classification: O31; G32; L60; L80.

MSC2010: 68T07.

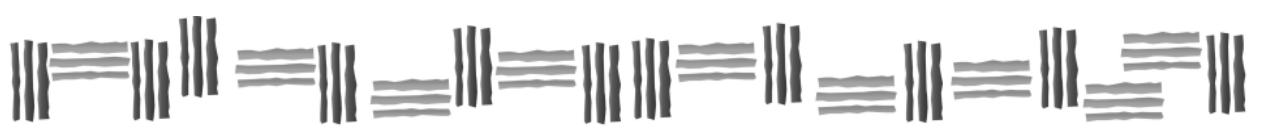




\section{Introducción}

El campo de la financiación de la innovación ha estudiado aisladamente o en pequeños grupos aspectos específicos de la financiación que influyen sobre el desarrollo de actividades de innovación en las empresas; aspectos tales como la estructura de capital (Chen, Hsu, \& Huang, 2010; O'Brien, 2003; Serrasqueiro, Nunes, \& da Rocha Armada, 2016), los obstáculos financieros a la innovación (Czarnitzki, 2006; Czarnitzki, Hottenrott, \& Thorwarth, 2011; Mohnen, Palm, van der Loeff, \& Tiwari, 2008) o el uso de las fuentes de financiación para la innovación (Beaudry \& Allaoui, 2012; Brancati, 2015; Brown, Fazzari, \& Petersen, 2009; Mancusi \& Vezzulli, 2014). Múltiples modelos teóricos, incluso contradictorios entre sí, han sido utilizados para describir el campo (Padilla-Ospina, MedinaVásquez, \& Rivera-Godoy, 2018).

Incluso si se considera toda la economía de la innovación como campo abarcador, este se caracteriza por una fragmentación teórica y metodológica, con enfoques tan diferentes como los de la macroeconomía, la organización industrial, las finanzas públicas y el desarrollo económico (Hall \& Rosenberg, 2010, p. 3-5). Esta fragmentación ha impedido que se pueda tener un panorama global del impacto sobre la intensidad de la innovación de las diferentes variables asociadas a la financiación en un solo modelo empírico.

Existe así un vacío en la literatura empírica, dado que no se ha planteado previamente un único modelo comprehensivo de la importancia relativa de las variables financieras para intensificar las actividades de innovación en el que se identifique qué efecto tiene cada una de estas variables financieras. Si bien Padilla-Ospina, Medina-Vásquez y Ospina-Holguín (2021) ofrecieron un modelo para la presencia o ausencia de la innovación, el modelo que se presenta en este artículo predice directamente la intensidad de la innovación e incluye las veintidós subvariables financieras independientes y las seis subvariables de control de Padilla-Ospina et al. (2021), además de dieciséis nuevas subvariables financieras independientes.

El presente trabajo desarrolla primero un modelo comprehensivo no necesariamente lineal para predecir la intensidad de la innovación a partir de los factores asociados a la financiación de la innovación. La variable que describe la intensidad de la innovación incluye tanto a las entradas como a las salidas de la innovación. Los factores asociados a la financiación de la innovación comprenden casi veinte variables generales, que cubren desde las características para acceder al crédito, las características del crédito, las fuentes de financiación a corto y largo plazo, las preferencias por las fuentes de financiación y la dificultad de obtener financiación a partir de estas fuentes, el riesgo moral y los costos de agencia, la aversión al riesgo del gerente, los costos de ajuste, de quiebra y de capital, y variables financieras asociadas específicamente a los proyectos de innovación.

El modelo predictivo se basa en un conjunto de dieciséis redes neuronales optimizadas cada una mediante optimización bayesiana. El modelo se construyó en una muestra estratificada por ciudad, tamaño, sector y presencia de la innovación de 157 pymes colombianas y se validó en una segunda muestra de prueba, obtenida por conveniencia, de 82 pymes. En los datos de validación, el modelo logra un coeficiente de determinación $R^{2}$ fuera de la muestra por encima de 0.5 o 0.6 , según la definición utilizada del coeficiente. Posteriormente, como resultado del conjunto de modelos, se pudo calcular una jerarquía de las variables que más importancia tienen para predecir la intensidad de la innovación, así como los efectos, no necesariamente lineales, que tiene cada variable sobre la intensidad de la innovación.

Los resultados sugieren que las variables del costo de ajuste para modificar la producción, el tamaño por número de empleados (una variable de control), la aversión al riesgo del gerente, y los costos esperados de quiebra tanto generales, como asociados a la innovación, son los factores que más influyen en que las pymes colombianas intensifiquen su desarrollo de actividades de innovación. Le siguen las características del crédito, un grupo de obstáculos para innovar y las fuentes de financiación de corto plazo. 
Entre estas variables principales, cuatro variables, los costos de ajuste y el costo esperado de quiebra por innovación, el tamaño por empleados y la aversión al riesgo del gerente, tienen un efecto negativo en la intensificación de la innovación; mientras que tres variables, el costo esperado de quiebra general, las características del crédito y la fuente de financiación en el corto plazo, tienen un efecto positivo.

Este artículo se encuentra organizado de la siguiente manera. En primer lugar, se realiza una revisión de la literatura; después, se expone brevemente la metodología utilizada para la realización de la investigación; más adelante, se presentan los resultados y su discusión; y en las últimas dos secciones se presentan las conclusiones y los detalles del apéndice en línea.

\section{Marco teórico}

En el campo de la financiación de la innovación confluyen diversas teorías: por ejemplo, la teoría del costo de la agencia (Jensen \& Meckling, 1976), la teoría del riesgo moral (Holmstrom, 1989), el concepto del costo del capital (Lintner, 1965; Mossin, 1966; Ross, 1976; Sharpe, 1964; Treynor, 1961) y diferentes modelos de estructura de capital (Berger \& Udell, 1998; Modigliani \& Miller, 1958; Myers, 1984) han sido utilizados para describir distintos aspectos parciales del mismo fenómeno de la financiación de la innovación.

A veces, tales modelos se contradicen entre sí, como en el caso de las distintas teorías de la estructura de capital. Debido a que no hay una teoría unificada sobre la financiación de la innovación y a que los estudios existentes se enfocan en una o en un par de variables financieras bajo las teorías antes descritas, no existen estudios directamente comparables frente a la propuesta que se plantea en esta investigación.

El trabajo más similar es el de Padilla-Ospina et al. (2021), quienes se enfocan en modelar la presencia o ausencia de innovación, en lugar de la intensidad de la innovación. Sin embargo, existe evidencia empírica previa sobre una buena parte de las variables que se utilizaron en el modelo de predicción que aquí se plantea. A continuación, se presentan resultados sobre algunas de las principales variables tratadas en este estudio.

Una de las variables que más se ha estudiado en el campo de la financiación de la innovación es el uso de las fuentes de financiación para la innovación. Esta variable se relaciona con el tema de la estructura de capital. Algunos estudios encuentran que las empresas innovadoras utilizan deuda externa (Hahn, Minola, Vismara, \& De Stasio, 2019; Hottenrott \& Richstein, 2020; Howell, 2017; Wang, Li \& Furman, 2017), otros estudios consideran que las empresas innovadoras prefieren utilizar fondos internos para financiar sus actividades (Barona-Zuluaga \& Rivera-Godoy, 2017; Brown, Martinsson, \& Petersen, 2012), y otros, a su vez, sugieren que las empresas innovadoras se deberían financiar con la emisión de acciones (Brown et al., 2009; Guariglia \& Liu, 2014).

Los obstáculos para el desarrollo de actividades de innovación conforman otro factor ampliamente estudiado. Algunos estudios coinciden en que los obstáculos que más se presentan para el desarrollo de actividades de innovación son los altos costos del acceso a la financiación y de innovar, la falta de asociacionismo empresarial, la introducción de nuevas tecnologías por parte de la competencia, la carencia de pensamiento estratégico por parte del gerente, la falta de ayuda por parte del gobierno y la rigidez organizacional (García-Quevedo, Segarra-Blasco, \& Teruel, 2018; Merz, 2019; Nor, Bhuiyan, Said \& Alam, 2016; Zhang \& Zheng, 2020).

El costo de ajuste, por su lado, también ha sido estudiado. El costo de ajuste mide los costos de modificar aquellas variables que controla la empresa durante su producción (Farlex Financial Dictionary, 2012). El estudio de Xu (2020) sugiere que los costos de ajuste son mayores en las empresas innovadoras. Contratar nuevo personal asociado a la innovación, o reentrenar o despedir este tipo de 
personal, implica grandes costos de ajuste (Hall \& Lerner, 2010), ya que tal personal no se encuentra fácilmente en el mercado (Guariglia \& Liu, 2014) y al despedirlo este puede llevarse consigo a otras empresas parte de los frutos de la innovación desarrollada (Himmelberg \& Petersen, 1994).

Los costos de bancarrota también han sido explorados en las actividades de innovación. Estos pueden surgir del hecho de que los activos de un proyecto de innovación suelen ser difícilmente liquidables. Asimismo, las empresas innovadoras con altos niveles de deuda tienen más probabilidad de exhibir dificultades financieras (Moon, 2021), por lo que los bancos tenderán a no prestarles. Sin embargo, la evidencia sugiere que los costos de bancarrota pueden llegar a impulsar a las empresas deudoras a intensificar sus actividades de innovación (Acharya \& Subramanian, 2009; Higgins, 2012, p. 219).

En cuanto a la aversión al riesgo del gerente, se ha hallado evidencia que sugiere que entre mayor sea la actividad de innovación que se desarrolle en la empresa, menor será la aversión al riesgo del gerente (Ghadim, Pannell \& Burton, 2005; Moon, 2021).

El sector industrial también ha sido identificado como un importante factor que influye en la financiación de la innovación debido a las diferencias entre la rentabilidad y el riesgo de los sectores. Diversos estudios han establecido la correlación que existe entre el desarrollo de actividades de innovación en las empresas y el nivel de desarrollo de los sectores y la industria (Hsu, Tian \& Xu, 2014; Leiponen \& Poczter, 2014; Mazzucato, 2013).

En los estudios sobre la financiación de la innovación usualmente se utilizan como variables de control el tamaño y la edad de las pymes. En general, las empresas más pequeñas y más jóvenes tienden a tener mayores restricciones financieras (Álvarez \& Crespi, 2015; Cucculelli, 2018; Rivera-Godoy, 2015). En concordancia con esto, el estudio de Fernández (2017) halla un efecto positivo y significativo entre el tamaño (y la edad) y la probabilidad de innovar en las empresas latinoamericanas.

Otras variables de control que se tuvieron en cuenta en este trabajo fueron el perfil del gerente, el nivel de educación de los empleados y las redes de cooperación. En cuanto al nivel de educación de los empleados existe evidencia que sugiere que entre mayor sea su grado de conocimiento o nivel educativo, mayores probabilidades hay de que los empleados le aporten ideas innovadoras a la empresa (Romijn \& Albaladejo, 2002).

Adicionalmente, la experiencia que ha tenido el gerente y su nivel educativo también influyen en que se desarrolle una cultura de innovación en las empresas (Romijn \& Albaladejo, 2002). Asimismo, el estudio de Landry (2002), por ejemplo, sugiere que a mayor participación de la empresa en las redes empresariales, académicas y sociales, mayor es la probabilidad de la empresa de intensificar las actividades de innovación.

En Colombia, no hay evidencia sobre estudios que identifiquen las variables financieras más importantes para predecir la intensidad de la innovación, con excepción del trabajo de Fernández (2017) que estima la probabilidad de innovar y el nivel de innovación en varios países de Suramérica utilizando una regresión logística a partir de un número de variables seleccionadas. En general, los demás estudios que existen son de tipo descriptivo, tal como el de Sierra et al. (2009), quienes sugieren que las grandes empresas son las que más desarrollan actividades de innovación.

Por otra parte, otros estudios describen las principales fuentes de financiación que utilizan las empresas innovadoras colombianas y apuntan a que, en primera instancia, se utilizan los fondos internos, seguido de las fuentes externas (Barona-Zuluaga \& Rivera-Godoy, 2017; Barona-Zuluaga, Rivera-Godoy, Aguilera-Cifuentes, \& Garizado-Román, 2015; García-Pérez de Lema, Barona-Zuluaga, \& Madrid-Guijarro, 2013; Padilla-Ospina, Rivera-Godoy, \& Ospina-Holguín, 2015; Rivera-Godoy, 2015). Igualmente, se ha establecido que en Colombia el desarrollo de actividades de innovación es más común en los sectores manufacturero y de servicios (Barona-Zuluaga \& Rivera-Godoy, 2017). 


\section{Metodología}

El enfoque de estudio de esta investigación fue cuantitativo, centrado en el uso de herramientas de la analítica predictiva. La definición de innovación utilizada se refiere a la establecida por el Manual de Oslo, $3^{\text {a }}$ edición (OECD/Eurostat, 2005) que indica que la innovación es "la introducción de un nuevo, o significativamente mejorado, producto (bien o servicio), proceso, nuevo método de comercialización o un nuevo método organizativo, en las prácticas internas de la empresa, la organización del lugar de trabajo o las relaciones exteriores" (p.56). Cabe resaltar, sin embargo, que esta investigación solo considera la innovación de producto y de proceso, lo que concuerda con la definición de innovación introducida posteriormente a la aplicación de las encuestas por el Manual de Oslo, $4^{\mathrm{a}}$ edición (OECD/Eurostat, 2018). De acuerdo con las Encuestas de Desarrollo e Innovación Tecnológica en la Industria Manufacturera y de Servicios (EDIT VIII y EDITS VI) realizadas en Colombia por el Departamento Administrativo Nacional de Estadísticas (DANE, 2017, 2018), estos son los dos tipos de innovación que más se desarrollan en las empresas colombianas. Adicionalmente, al referirnos a la financiación de la innovación apelamos a Hall (2010), quien aclara que "la inversión en innovación generalmente consiste en gastos de Investigación y Desarrollo (I+D), gastos de diseño y marketing para llevar un nuevo producto al mercado, inversión en el nuevo equipo de capital necesario e inversión en capacitación" (p. 2).

Para entrenar y validar los modelos de predicción de la intensidad de la innovación se seleccionó una muestra a través de un cuestionario por muestreo estratificado por ciudad, tamaño de empresa, sector (servicios/industria) y presencia/ausencia de innovación en la empresa. Un total de 157 pymes fueron encuestadas. Para probar la generalidad de los modelos predictivos se utilizó una muestra distinta a la usada para entrenar y validar los modelos. Esta segunda muestra fue tomada por conveniencia y contiene un total de 82 cuestionarios recolectados.

Para la definición de pyme a partir del tamaño de la empresa se utilizó la identificación de una Mipyme, a partir del total de activos, descrita en el artículo 2 de la Ley 905 de 2004 de carácter nacional y se usó el valor del salario mínimo mensual legal vigente del año 2016 (Banco de la República de Colombia, 2017).

La variable de la intensidad de la innovación se construyó con base en el índice compuesto de innovación desarrollado por Carayannis y Provance (2008), que incluye variables de salida y de entrada de la innovación. La variable dependiente de "Intensidad de la innovación" fue la primera componente principal del análisis de componentes principales de los rasgos de las salidas de la innovación (cantidad de innovaciones, tipo y grado de innovación, ventas esperadas por innovación) y de las entradas de la innovación (protección a la innovación, empleados tiempo completo en innovación y gasto en innovación).

Para la elaboración del cuestionario se tuvieron en cuenta las variables como el costo de ajuste, la aversión al riesgo del gerente, el costo esperado de quiebra por innovación, el costo esperado de quiebra, las características del crédito, los obstáculos para la innovación, las fuentes de financiación de corto plazo, la asimetría de la información sobre proyectos de innovación, las características de la empresa para acceder al crédito, la dificultad de las fuentes de financiación, las fuentes de financiación de largo plazo, el costo de capital, el riesgo moral y los costos de agencia, la preferencia por las fuentes de financiación, la financiación obtenida para la innovación, las redes de cooperación y el spillover. (El spillover se refiere en este trabajo a los beneficios que obtiene la empresa por absorber el conocimiento en innovación que ha sido creado por terceras partes, por ejemplo, por otras empresas, sin haber tenido que incurrir en los costos de innovación (Czarnitzki \& Hottenrott, 2010). Para la definición específica de la variable, consúltese el Apéndice en línea).

Como variables de control se incluyeron el sector, el tamaño de la empresa, la edad de la empresa, el perfil del gerente, el nivel de educación de los empleados y las redes de cooperación. Para aquellas variables que requerían de información contable adicional, esta se obtuvo de la base de datos EMIS (2017). 
La construcción de las variables dependientes e independientes se basó en el análisis de componentes principales, el promedio y el reajuste para lograr rasgos predictivos óptimos (véase la Tabla 1 para una enumeración de las variables y el Apéndice en línea, incluida la Tabla A, para una descripción completa de las definiciones y los métodos de construcción de las variables).

Tabla 1. Variables de control, independientes y dependientes.

\section{Variable dependiente \\ A. Intensidad de la} innovación:
A1. Salidas de la innovación:

A2. Entradas de la innovación:
A.1.1. Cantidad de innovaciones A.1.2. Tipo y grado de innovación A.1.3. Ventas esperadas por innovación A.2.1. Protección a la innovación A.2.2. Empleados tiempo completo en innovación A.2.3. Gasto en innovación

\section{Variables independientes}

A. Características para acceder al crédito:

A1. Acceso al crédito
A2. Garantía para la financiación:

A3. Falta de garantía anterior A4. Liquidez:

A5. Endeudamiento:

B. Características del crédito:

C. Fuentes de financiación a corto plazo

D. Fuentes de financiación a largo plazo

E. Preferencia por las fuentes de financiación

F. Dificultad de las fuentes de financiación

G. Riesgo moral y costos de agencia

H. Aversión al riesgo del gerente

I. Costos de ajuste

J. Costo esperado de quiebra

K. Costo de capital

L. Financiación obtenida para la innovación

M. Subsidios

N. Spillover

O. Deducción de impuestos por innovación

P. Costo esperado de quiebra por innovación
B1. Plazo del crédito

B2. Tasa de financiación
A2.1. Colateral

A2.2. Requirió garantía

A2.3. Tipo de garantía

A2.4. Monto de la garantía

A4.1. Promedio de la razón corriente y prueba ácida.

A4.2. Razón corriente

A4.3. Prueba ácida

A5.1. Apalancamiento

A5.2. Razón de endeudamiento del activo

A5.3. Apalancamiento a largo plazo 
Q. Asimetría de la información sobre proyectos de innovación

R. Obstáculos para la innovación 1:

S. Obstáculos para la innovación 2:
R1. Percepción de riesgo

económico

R2. Período de retorno de la inversión muy largo

R3. Saturación del mercado

R4. Falta de infraestructura o maquinaria

$\mathrm{R} 5$. Incertidumbre sobre introducción de innovación al mercado

R6. Falta de personal calificado

R7. Impacto negativo de regulaciones o leyes

$\mathrm{S} 1$. Costos directos para innovar elevados

S2. Costos de la financiación de la innovación muy altos

S3. Rigidez organizacional

S4. Falta de información sobre el

mercado

S5. Falta de información sobre

nuevas tecnologías

T. Redes de cooperación

\begin{tabular}{ll}
\hline Variables de control & \\
\hline A. Perfil del gerente: & A1. Educación del gerente \\
& A2. Experiencia del gerente \\
B. Tamaño por empleados & \\
C. Educación de los & \\
D. Edad de la empresa & \\
F. Sector mayor & \\
\hline
\end{tabular}

Nota: Para una descripción del proceso de ingeniería de rasgos, las definiciones de cada variable y las fuentes en las que se inspiran, ver el Apéndice en línea. Las variables "Subsidios" y "Deducción de impuestos por innovación" no se tuvieron en cuenta en los modelos predictivos finales por ser de varianza casi cero (Kuhn \& Johnson, 2013).

Fuente: Elaboración propia basada en Padilla-Ospina et al. (2021).

Los modelos predictivos utilizados se basaron en redes neuronales. En el uso de la importancia de las variables a partir de redes neuronales este estudio es pionero, aunque previamente se habían utilizado modelos de redes neuronales para predecir el desempeño de la innovación, por ejemplo en las industrias de Taiwán (Chien, Wang, \& Lin, 2010; Wang \& Chien, 2006). Una red neuronal consta de variables que se organizan en capas enlazadas de manera similar a las conexiones de las neuronas de un cerebro (Ho, 2012). La predicción que resulta de una red neuronal está modelada por diversas variables intermedias o unidades ocultas y se pueden presentar redes neuronales de una o múltiples capas intermedias. En el modelo más básico de una red neuronal feedforward de una sola capa oculta, la capa inicial la conforman los predictores, que envían los datos de entrada a una capa intermedia con unidades ocultas. Cada una de estas unidades ocultas está, a su vez, conectada hacia adelante con la capa de salida, compuesta de una única unidad que calcula la variable de salida. En este modelo en particular, cada unidad oculta resulta de aplicar una función de activación a una combinación lineal de los predictores de la capa anterior y la unidad que determina la variable de salida, esto es, la intensidad de la innovación, es una combinación lineal de las unidades ocultas mencionadas (Kuhn \& Johnson, 2013; Titterington, 2010). 
Al calcular los modelos de redes neuronales se utilizó la optimización bayesiana con el fin de optimizar los parámetros que definen cada modelo alterando tanto el tipo de modelo (red convolucional o feedforward) como los conjuntos de hiperparámetros que definen cada modelo particular (número de conexiones, número de capas o función de activación, sea logística, rampa o tangente hiperbólica). La bondad o adecuación del modelo de regresión se calculó con la raíz del error cuadrático medio. Asimismo, se calculó el coeficiente de determinación $R^{2}$ de dos maneras distintas. Los valores que se reportan en este trabajo corresponden a la bondad o adecuación del modelo de regresión estimada en la muestra de prueba, es decir, en datos totalmente fuera de la muestra inicial utilizada para la construcción del modelo.

La raíz del error cuadrático medio se calcula como:

$$
R M S E=\left(\frac{1}{N} \sum_{i=1}^{N}\left(\hat{y}_{i}-y_{i}\right)^{2}\right)^{1 / 2}
$$

donde $\hat{y}_{i}$ es el valor predicho de la intensidad de la innovación en una empresa $i$-ésima fuera de la muestra, $y_{i}$ es el valor medido real de la intensidad de innovación en esa misma empresa y $N$ es el tamaño de los datos de prueba, es decir, 82. Un valor pequeño del RMSE indica que las predicciones fuera de la muestra están muy cercanas a los valores reales de intensidad de innovación.

La primera manera utilizada para calcular el coeficiente de determinación, que se denominará $R_{1}^{2}$, corresponde al cuadrado del coeficiente de correlación entre los valores observados y los valores predichos en los datos de prueba (Kuhn \& Johnson, 2013). La segunda manera de cálculo, que se denominará $R_{2}^{2}$, corresponde a la siguiente fórmula (Kvalseth, 1985):

$$
R_{2}^{2}=1-\frac{R M S E^{2}}{R M S E_{\text {base }}^{2}},
$$

donde

$$
R M S E_{\text {base }}=\left(\frac{1}{N} \sum_{i=1}^{N}\left(\bar{y}_{i}-y_{i}\right)^{2}\right)^{1 / 2}
$$

y $\bar{y}_{i}$ es el valor medio de la intensidad de la innovación en los datos de prueba, es decir,

$$
\bar{y}_{i}=\frac{1}{N} \sum_{i=1}^{N} y_{i}
$$

La primera manera de calcular el coeficiente de determinación evalúa lo correlacionados que están los valores reales de la variable dependiente con los valores predichos fuera de la muestra. A su vez, la segunda manera de calcular el coeficiente de determinación evalúa fuera de la muestra la mejora en el error cuadrático medio del modelo de regresión particular respecto al modelo ingenuo donde cada predicción es el valor promedio de los datos. Ambos coeficientes de determinación pueden tomar valores entre 0 y 1 , siendo 1 el valor óptimo.

Para obtener la importancia de la variable o rasgo $j$ de acuerdo con el modelo predictivo dado $k$, la importancia de la variable $j$ se calcula como el cociente del error cuadrático medio del modelo $k$ una vez se permutan aleatoriamente entre las empresas los valores de la variable $j$ en cuestión (en la muestra de prueba) sobre el error cuadrático medio del modelo $k$ de base (sin cambios) (Molnar, 2020). 
La media, desviación estándar y en general la distribución de la importancia de la variable $j$ para un modelo $k$ es simplemente la media, desviación estándar y distribución empírica que resulta de repetir este proceso $M$ veces (en este trabajo $M=50$ ). De acuerdo con este procedimiento, una variable es más importante cuando la permutación de sus valores deteriora más el error de predicción del modelo original.

Finalmente, para calcular el efecto de las variables financieras y de control en la intensidad de la innovación de las pymes colombianas se elaboró un gráfico marginal de cada una de las variables calculadas en el modelo de predicción. Los gráficos marginales estiman el efecto y la dirección del efecto de cada variable en la empresa "promedio", definida esta como aquella que tiene como valor de cada variable la mediana de esta variable en la muestra que representa la población. Para construirlos se calculan los valores medianos para los factores predictivos para las empresas en la muestra de entrenamiento y se evalúa cómo cambia la intensidad de la innovación de acuerdo con el modelo de predicción con un cambio en la variable particular estudiada cuando las demás variables toman estos valores medianos ceteris paribus.

\section{Resultados}

A continuación, se presentan los resultados en términos de la jerarquía de las variables según su importancia para predecir la intensidad de la innovación y, más adelante, se presentan los efectos de cada variable sobre la intensidad de la innovación.

\subsection{Importancia de las variables financieras para la intensidad de la innovación}

Después de experimentos preliminares, para construir los modelos de predicción se realizó una búsqueda grid del número de conexiones, yendo desde una red neuronal de 50 conexiones a una de 125 conexiones, en intervalos de cinco en cinco. Es decir, para un número de conexiones establecido, se realizó la optimización bayesiana de los demás hiperparámetros en cada caso. El máximo de rondas de entrenamiento fue de $4000 \mathrm{y}$ los modelos fueron entrenados en unidades de procesamiento gráficos (GPUs), (excepto para el modelo de 60 parámetros donde se usaron 3000 rondas, ya que con 4000 rondas se convergía a un modelo anómalo).

A los modelos óptimos se les impuso un parámetro de regularización igual a $(m-50) / 25$, donde $m$ es el número de conexiones de la red neuronal con el propósito de evitar el sobreajuste que se halló experimentalmente al aumentar el número de parámetros. Hubo una amplia diversidad de modelos resultantes, de acuerdo con la selección realizada por la optimización bayesiana de los demás hiperparámetros para cada número de conexiones. Algunos modelos fueron redes totalmente conectadas y otros, redes convolucionales; distintos modelos utilizaron diferentes funciones de activación, según el caso, a saber, la función logística, la función tangente hiperbólica y la función rampa; y el parámetro de dropout estuvo entre 0.21 y 0.70 , bajo optimización Adam. Adam es un algoritmo de optimización que se puede utilizar en lugar del algoritmo de descenso de gradiente estocástico clásico. Adam actualiza los pesos de la red neuronal de forma iterativa a partir de tasas de aprendizaje para cada parámetro basadas en un promedio móvil exponencial del gradiente y del gradiente al cuadrado (Kingma \& Ba, 2017). La profundidad de la red (el número de capas ocultas) seleccionada por la optimización bayesiana fue, sin embargo, consistentemente de dos, por lo que se privilegiaron redes no muy profundas.

En la Tabla 2 se puede apreciar la bondad o adecuación de cada uno de los dieciséis modelos de redes neuronales en los datos de prueba, es decir, totalmente fuera de la muestra. De manera general, se puede establecer que los dieciséis modelos tienen buenos resultados en términos del $R S M E$ y del $R_{1}^{2}$ y $R_{2}^{2}$. Debe recordarse cómo en las regresiones de redes neuronales se espera que un $R M S E$ sea bajo, lo 
que sugiere que las predicciones por fuera de la muestra están muy cercanas a los valores reales de la intensidad de la innovación; mientras que se esperan valores cercanos a 1 de $R_{1}^{2}$ y $R_{2}^{2}$.

Tabla 2. Resumen de los valores de bondad de ajuste de los dieciséis modelos de redes neuronales.

\begin{tabular}{cccc}
\hline $\begin{array}{c}\text { Número de } \\
\text { Parámetros }\end{array}$ & $\mathbf{R M S E}$ & $\mathbf{R}_{\mathbf{2}}^{\mathbf{1}}$ & $\mathbf{R}_{\mathbf{2}}^{\mathbf{2}}$ \\
\hline $\mathbf{5 0}$ & 0.30 & 0.59 & 0.53 \\
\hline $\mathbf{5 5}$ & 0.29 & 0.58 & 0.55 \\
\hline $\mathbf{6 0}$ & 0.34 & 0.58 & 0.38 \\
\hline $\mathbf{6 5}$ & 0.32 & 0.48 & 0.46 \\
\hline $\mathbf{7 0}$ & 0.29 & 0.60 & 0.54 \\
\hline $\mathbf{7 5}$ & 0.29 & 0.56 & 0.54 \\
\hline $\mathbf{8 0}$ & 0.28 & 0.60 & 0.58 \\
\hline $\mathbf{8 5}$ & 0.32 & 0.58 & 0.44 \\
\hline $\mathbf{9 0}$ & 0.31 & 0.60 & 0.49 \\
\hline $\mathbf{9 5}$ & 0.32 & 0.63 & 0.45 \\
\hline $\mathbf{1 0 0}$ & 0.34 & 0.72 & 0.37 \\
\hline $\mathbf{1 0 5}$ & 0.33 & 0.56 & 0.42 \\
\hline $\mathbf{1 1 0}$ & 0.32 & 0.53 & 0.45 \\
\hline $\mathbf{1 1 5}$ & 0.30 & 0.67 & 0.52 \\
\hline $\mathbf{1 2 0}$ & 0.33 & 0.46 & 0.40 \\
\hline $\mathbf{1 2 5}$ & 0.39 & 0.33 & 0.21 \\
\hline
\end{tabular}

Nota: Los modelos con 70, 80 y 115 conexiones son los de mejor desempeño general en la predicción de la intensidad de innovación. El RMSE denota la raíz del error cuadrático medio y los coeficientes de determinación fuera de la muestra $R_{1}^{2}$ y $R_{2}^{2}$ se calculan de acuerdo con lo referido en la sección de Metodología.

Fuente: Elaboración propia.

En general, en los modelos con 70, 80 y 115 conexiones se presentaron los valores del RMSE más bajos y los valores más altos del $R_{1}^{2}$ y $R_{2}^{2}$. El modelo de 80 parámetros es el mejor de los dieciséis modelos realizados, con un $R S M E$ de 0.28 , un $R_{1}^{2}$ de 0.60 y un $R_{2}^{2}$ de 0.58 . El modelo de 70 parámetros es el segundo mejor modelo con un $R S M E$ de 0.29 , un $R_{1}^{2}$ de 0.60 y un $R_{2}^{2}$ de 0.54 . El tercer mejor modelo es el de 115 conexiones con un RMSE de 0.30 , un $R_{1}^{2}$ de 0.67 y un $R_{2}^{2}$ de 0.52 .

En la Figura 1 se pueden apreciar los resultados del modelo que surge de la combinación de los dieciséis modelos de regresión de redes neuronales para predecir la intensidad de la innovación. En él se observa un $R S M E$ de 0.29 , un $R_{1}^{2}$ de 0.63 y un $R_{2}^{2}$ de 0.55 , siendo un modelo bastante robusto fuera de la muestra, aunque no mejor que los modelos de 70, 80 y 115 parámetros. Por esta razón, no se calculó la importancia de las variables en este modelo combinado, recurriéndose mejor al gráfico de rankings relativos de la importancia de las variables para un panorama general de la importancia de las variables en todos los dieciséis modelos. 
Figura 1. Gráfico de residuos del modelo combinado de los dieciséis modelos de redes neuronales de predicción de la intensidad de innovación.

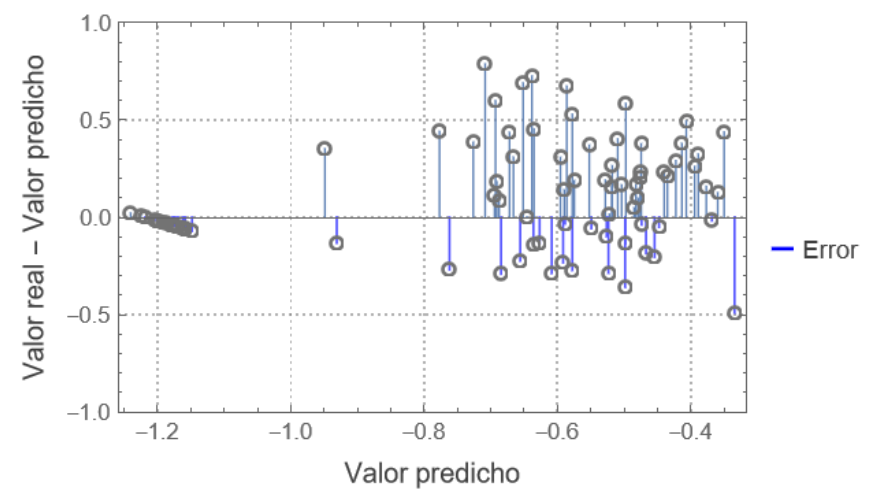

Fuente: Elaboración propia.

En la Figura 2 se indica la posición en el ranking de importancia de las variables de cada variable independiente en cada uno de los dieciséis modelos de redes neuronales que predicen la intensidad de la innovación.

Figura 2. Rankings de importancia relativa de las variables para cada modelo de red neuronal de predicción de la intensidad de la innovación.

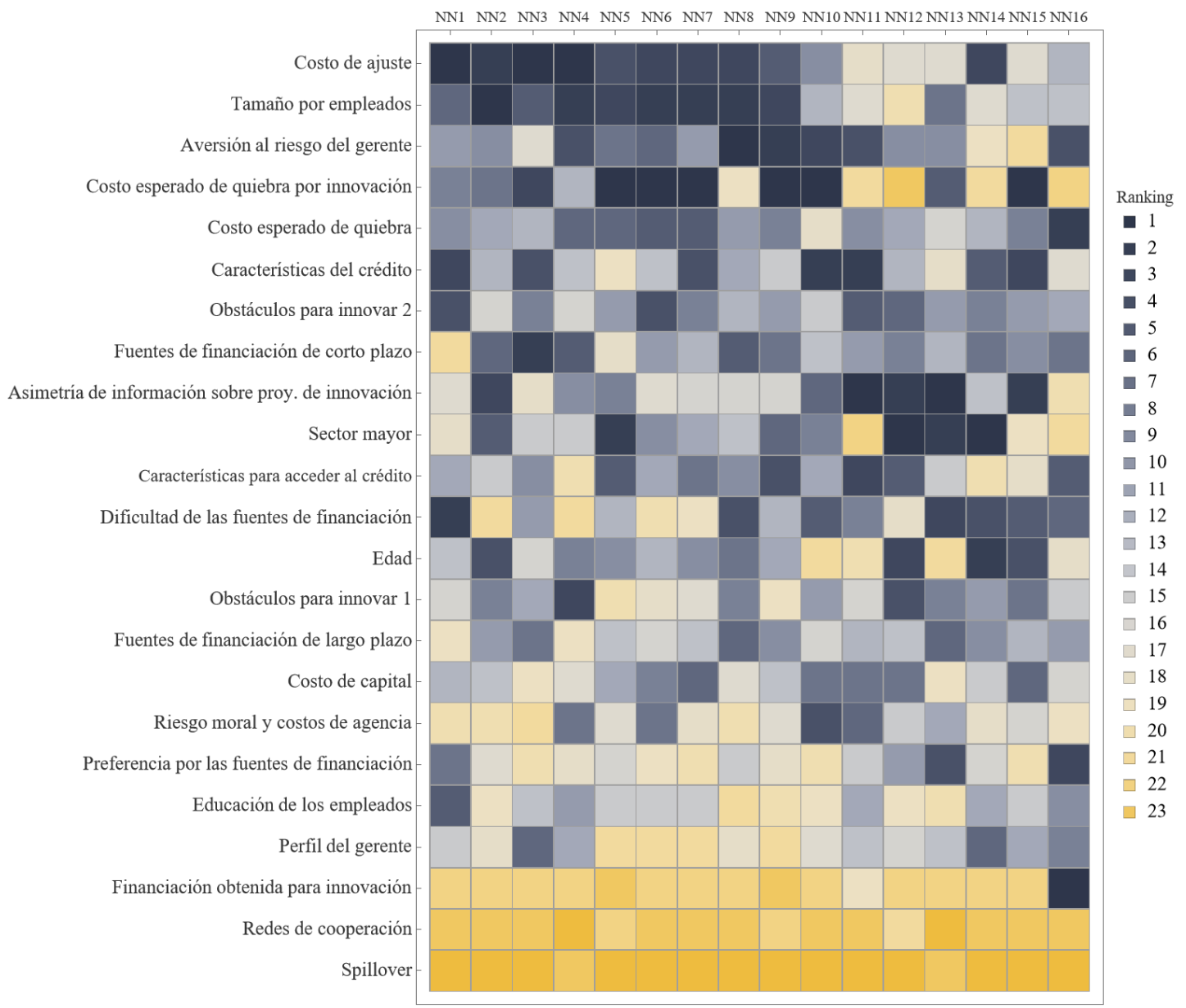

Fuente: Elaboración propia. 
Las variables independientes a la izquierda de la Figura 2 aparecen ordenadas por el ranking promedio de cada variable en los dieciséis modelos: en la escala del ranking, 1 corresponde a la variable más importante y 23 a la menos importante.

Las nueve variables más importantes para la intensidad de la innovación fueron: "Costo de ajuste", "Tamaño por empleados", "Aversión al riesgo del gerente", "Costo esperado de quiebra por innovación", "Costo esperado de quiebra", "Características del crédito", "Obstáculos para innovar 2", "Fuentes de financiación de corto plazo" y "Asimetría de información sobre proyectos de innovación". Entre estas primeras nueve variables, la segunda fue una variable de control: "Tamaño por empleados", variable que resultó en principio más importante que las demás variables financieras, con la excepción del "Costo de ajuste".

\subsection{Efecto de las variables financieras en la intensidad de la innovación en las pymes colombianas}

A través de los gráficos marginales de las dieciséis variables del modelo, se calculó el efecto aproximado de cada variable sobre la intensidad de innovación en la empresa "promedio". Específicamente, el gráfico marginal muestra cómo cambia la intensidad de innovación al cambiar la variable en cuestión ceteris paribus asignando a todas las demás variables su mediana respectiva en la muestra de entrenamiento.

Entre las variables que tienen un efecto positivo en la intensidad de la innovación, es decir, aquellas en las que a mayor valor de la variable, mayor es la intensidad de la innovación, se encuentran: "Educación de los empleados", "Edad de la empresa", "Sector mayor", "Redes de cooperación", "Spillover", "Costo esperado de quiebra", "Perfil del gerente", "Fuentes de financiación a corto plazo", "Preferencia por las fuentes de financiación", "Características del crédito" y "Financiación obtenida para innovación". Al contrario, las variables que afectan negativamente la intensidad de las actividades de la innovación son: "Tamaño por empleados", "Aversión al riesgo del gerente", "Riesgo moral y costos de agencia", "Costo de ajuste", "Fuentes de financiación de largo plazo", "Dificultad de las fuentes de financiación", "Características para acceder al crédito", "Costo esperado de quiebra por innovación" y "Obstáculos para innovar 1". Las variables que en apariencia no tuvieron un efecto muy apreciable en la intensidad de la innovación son el "Costo de capital" y "Obstáculos para innovar 2".

El gráfico marginal de la variable "Asimetría de información sobre proyectos de innovación" exhibe una forma de U, siendo este un hallazgo nuevo para este tipo de estudios. Gráficos marginales similares resultaron al utilizar un modelo combinado de predicción a partir de los tres mejores modelos de redes neuronales. Las siguientes variables tuvieron un efecto diferente en este nuevo modelo combinado: "Edad" (efecto no muy apreciable), "Aversión al riesgo del gerente" (efecto positivo), "Costo de capital" (efecto positivo), "Perfil del gerente" (efecto negativo), "Fuentes de financiación de corto plazo" (efecto ligeramente negativo), "Asimetría de información sobre proyectos de innovación" (efecto negativo), "Obstáculos para innovar 1" (efecto ligeramente positivo), "Obstáculos para innovar 2" (efecto positivo).

\section{Discusión}

A continuación, se discuten brevemente los resultados de las diez variables más importantes para que las pymes colombianas intensifiquen sus actividades de innovación de acuerdo con los resultados obtenidos a partir de la predicción de los dieciséis modelos de redes neuronales y después de identificar las variables más importantes y su efecto.

Las variables "Costo de ajuste" y "Aversión al riesgo del gerente" son las dos más importantes (con excepción de la variable de control "Tamaño por empleados") para la predicción de la intensidad de la innovación. El valor reajustado promedio para las empresas que innovan del "Costo de ajuste" es de 0.04 , mientras que es de 0.03 para las empresas que no innovan, es decir, es menor cualitativamente 
para las empresas que no innovan (empero, la diferencia no es significativa de acuerdo con el test de Mann-Whitney). Lo hallado en este trabajo sugiere que las pymes que innovan tienen una mayor dificultad para modificar su producción en consonancia con las nuevas innovaciones, pero, a pesar de esa mayor dificultad, deciden innovar. El gráfico marginal de esta variable evidencia un efecto negativo sobre la intensidad de la innovación, es decir, a mayor costo de ajuste menor es la intensidad de las actividades de innovación en la empresa que tiene como valores en las demás variables la mediana. Otros estudios ya habían encontrado que los costos de ajuste eran más altos para las empresas que innovan (Hall \& Rosenberg, 2010; Xu, 2020), pero la importancia fundamental de esta variable había sido ignorada hasta ahora.

El promedio de la variable reajustada "Aversión al riesgo del gerente" es mucho mayor en las empresas que innovan $\left(0.08^{* * *}\right)$ que en las que no innovan $(-0.01)$, de acuerdo con el test de MannWhitney (donde los tres asteriscos denotan que la variable es mayor con significancia estadística al 1\%). Este resultado sugiere que los gerentes de las empresas innovadoras son más aversos al riesgo que los gerentes de las empresas que no innovan, lo que indica que emprenden procesos de innovación, considerados típicamente como de alto riesgo, de una manera hipotéticamente más planificada y racional que impulsiva y arriesgada. De acuerdo con el gráfico marginal, al aumentar la aversión al riesgo del gerente, la intensidad de la innovación disminuye en el modelo de predicción combinado principal. Este resultado concuerda con lo encontrado por Tsur, Sternberg y Hochman (1990) y Ghadim, Pannell y Burton (2005), quienes sugieren que una mayor aversión al riesgo del gerente reduce el desarrollo de actividades de innovación en las empresas.

En cuanto a la segunda variable más importante en el ranking promedio de los dieciséis modelos de redes neuronales, el "Tamaño por empleados", las empresas que innovan son significativamente más grandes, con $90.15^{* * *}$ empleados en promedio, que las empresas que no innovan, con 50.34 empleados en promedio, (donde tres asteriscos denotan que la variable de la pareja es mayor con significancia estadística al 1\% según el test de Mann-Whitney). Sin embargo, de acuerdo con el gráfico marginal, a mayor tamaño de la empresa por número de empleados la intensidad de la innovación disminuye, lo que sugiere que puede que las empresas innovadoras tiendan a ser más grandes, como lo ha hallado por ejemplo Langebaek y Vásquez (2007) y Fernández (2017), pero que son las pequeñas y medianas empresas las que exhiben mayor intensidad de innovación.

Las variables que siguen en el ranking promedio de importancia de las variables para la predicción de la intensidad de la innovación se relacionan precisamente con el riesgo y son el "Costo esperado de quiebra por innovación" y el "Costo esperado de quiebra". El "Costo esperado de quiebra" no difiere estadísticamente entre las empresas que innovan y las que no innovan, pero cuando se remueven los valores en el $10 \%$ menor y mayor para calcular una media truncada, se puede apreciar que el costo esperado de quiebra es mayor en las empresas que innovan $\left(1003.2^{* *}\right.$ millones de pesos colombianos) que en las que no innovan (502 millones de pesos), de acuerdo con el test de MannWhitney (donde dos asteriscos denotan que la variable de la pareja es mayor con significancia estadística al 5\%). El gráfico marginal de la empresa "promedio" evidencia que la intensidad de la innovación aumenta al aumentar el costo esperado de quiebra. Esto coincide con por lo menos dos estudios que confirman que los costos de bancarrota impulsan a las empresas deudoras a desarrollar más actividades de innovación (Acharya \& Subramanian, 2009; Higgins, 2012; Moon, 2021).

Por otro lado, el "Costo esperado de quiebra por innovación" antes del reajuste solo se puede medir finitamente en las empresas que innovan (por definición fue ilimitado en las empresas que no innovan, lo que es tratado adecuadamente por la función de reajuste en el rasgo final). El costo esperado de quiebra promedio por innovación fue de 2444.5 millones de pesos colombianos, exceptuando una empresa para la cual se designó como ilimitado. El gráfico marginal sugiere que entre mayor sea el costo de quiebra por innovación menor es la intensidad de las actividades de innovación. Este hallazgo coincide con la evidencia presentada, por ejemplo, por Aghion, Bloom, Blundell, Griffith y Howitt (2002). 
La variable "Características del crédito" muestra que la tasa de los créditos a los que accedieron las empresas que innovan respecto a las que no innovan no difiere estadísticamente, según el test de Mann-Whitney, aunque es cualitativamente mayor en un punto básico. También muestra que el plazo del crédito sí resulta significativamente mayor para las empresas que innovan respecto a las que no innovan, según la misma prueba. El gráfico marginal demuestra que existe un efecto positivo de las "Características del crédito" con la probabilidad de intensificar las actividades de innovación. De acuerdo con esto, una tasa de crédito y un plazo favorable ayuda a que la empresa intensifique sus actividades de innovación. En la literatura, algunos estudios evidencian que las altas tasas de interés en los créditos para las pymes innovadoras tienen un impacto negativo en el desarrollo de actividades de innovación (Botta, 2017; Kleinknecht, 2016; Lussuamo \& Serrasqueiro, 2020), por lo que Menshchikova y Sayapin (2016) y Merz (2019), por ejemplo, sugieren la implementación de políticas públicas que permitan brindar a las pymes innovadoras créditos con tasas de intereses más bajas y con plazos de pago mayores para incentivar el desarrollo de la innovación.

Aunque se encuentra en la séptima posición del ranking derivado de la predicción de la intensidad de la innovación, vale la pena discutir la variable "Obstáculos para la innovación 2". Las variables "Obstáculos para la innovación 1" y "2" corresponden a las dos componentes principales reajustadas del análisis de componentes principales de la relevancia de los obstáculos para la innovación calculada a partir de una escala de Likert sobre la relevancia de un listado de obstáculos (ver Tabla A en el Apéndice en línea).

La primera componente principal tiene cargas mayores a 0.4 (en valor absoluto) en los obstáculos: percepción de un excesivo riesgo económico, un periodo del retorno de la inversión demasiado largo, demasiada incertidumbre sobre la introducción de la innovación al mercado, costos directos de innovar demasiado altos, costos de la financiación de la innovación demasiado altos, falta de disponibilidad de financiación para innovar, falta de infraestructura o maquinaria, falta de personal calificado, falta de información sobre nuevas tecnologías, falta de información sobre las características del mercado, rigidez organizacional dentro de la empresa e impacto negativo de regulaciones o leyes. Por otro lado, la segunda componente principal que corresponde a "Obstáculos para la innovación 2" se concentra en los costos directos de innovar demasiado altos, costos de la financiación de la innovación demasiado altos, falta de información sobre nuevas tecnologías, falta de información sobre las características del mercado y rigidez organizacional dentro de la empresa.

Al examinar la variable reajustada se deduce que las empresas que innovan tienen cualitativamente menores obstáculos del tipo que corresponden a "Obstáculos para la innovación 2" (con un valor de la variable medio de -0.004) respecto a las empresas que no innovan (0.024). Aunque la diferencia no es significativa de acuerdo con el test $t$ de Student, la diferencia cualitativa sugeriría que las empresas que innovan tienen, hipotéticamente, de acuerdo con las cargas de la componente principal, costos más bajos de innovar, menos información sobre la innovación y más rigidez organizacional en promedio (teniendo en cuenta los signos de las cargas).

Estos resultados concuerdan con algunos estudios que sugieren que los obstáculos a la innovación más relevantes para las empresas que innovan están relacionados con los factores de costos y conocimiento (Galia, Mancini \& Morandi, 2012; García-Quevedo et al., 2018; Zhu, Wittmann \& Peng, 2012). Por otra parte, autores como Damanpour (1991) y Galia y Legros (2004) han hallado que la rigidez organizacional es otro factor que puede obstaculizar el desarrollo de actividades de innovación. Sin embargo, cabe destacar que la variable "Obstáculos para innovar 2" parece no tener mayor efecto para la intensificación de la innovación según el gráfico marginal del modelo combinado principal (o tiene un efecto positivo, según el modelo combinado que utiliza las mejores tres redes neuronales).

En cuanto a la variable "Fuentes de financiación de corto plazo", se deduce que las empresas, innoven o no, utilizan más fondos internos o utilidades retenidas y luego préstamos bancarios o crédito de proveedores o clientes. El gráfico marginal sugiere que, a mayor uso de fuentes de financiación en el corto plazo, mayor es la intensidad de las actividades de innovación en la empresa. Esto coincide con los resultados de Ghisetti y Montresor (2020), por ejemplo. Los trabajos de Barona-Zuluaga, Rivera- 
Godoy y Aguilera-Cifuentes (2015) y Barona-Zuluaga y Rivera-Godoy (2017) de hecho sugieren que las empresas colombianas innovadoras financian sus actividades de innovación especialmente con recursos internos, financiación bancaria y créditos de proveedores, lo que coincide con lo obtenido en el presente estudio.

La novena variable que se registra en el ranking de importancia de las variables para la predicción de la intensidad de la innovación es la "Asimetría de información sobre proyectos de innovación". Usando la escala de Likert de 1 a 5 antes del reajuste, donde 1 denota poca asimetría de información y 5 mucha, se puede concluir que las empresas que innovan (con un promedio de 3.95) no difieren significativamente en esta variable respecto a las empresas que no innovan (con un promedio de 3.78), según el test $t$ de Student.

Existen dos puntos de vista en la literatura a este respecto. Autores como Aboody y Lev (2000) consideran que las actividades de I+D mejoran los problemas de asimetría de información en las empresas; pero, al contrario, estudios como los de Markman, Balkin y Schjoedt (2001) y Canepa y Stoneman (2007) sugieren que los problemas de asimetría de la información son severos en las pymes innovadoras. Precisamente, en concordancia con esta paradoja, el gráfico marginal de la empresa "promedio" muestra que el efecto de la variable "Asimetría de información sobre proyectos de innovación" sobre la intensidad de la innovación de la empresa "promedio" tiene forma de U y es, por ende, no lineal, lo que explicaría que se puedan hallar tanto relaciones positivas como negativas de la asimetría con la intensidad de la innovación, dependiendo del nivel de asimetría presente.

La variable "Sector mayor" resulta ser de importancia intermedia. En cuanto a la diferencia en el sector mayor, el $46.32 \%$ de las empresas que innovan pertenecen al sector industrial, mientras que el $42.37 \%$ de las empresas que no innovan pertenecen al sector de servicios, sin que haya una diferencia significativa de acuerdo con el test Chi cuadrado $N-1$ (Campbell, 2007). En el gráfico marginal se demuestra que el "Sector mayor" tiene un efecto positivo sobre la intensidad de la innovación, indicando que al pasar una empresa del sector de manufactura al de servicios se esperaría una mayor intensidad en las actividades de innovación. Esto coincide con autores como Leiponen y Poczter (2014) y Hsu et al. (2014) que encuentran que el sector influye en el desarrollo de las actividades de innovación.

En suma, los resultados de este trabajo arrojan un nuevo y amplio panorama sobre la financiación de la innovación que permite afirmar que para que las pymes decidan intensificar sus actividades de innovación predominan las variables capaces de limitar la innovación, como el costo de ajuste y algunos obstáculos para innovar, pero sobre todo predominan las variables centradas en el riesgo de quiebra de la empresa o del proyecto de innovación y en la aversión al riesgo del gerente. De esta manera, se llena un vacío en la literatura respecto a cuáles son las variables asociadas a la financiación de la innovación que más influyen en la intensificación de las actividades de innovación, en este caso en un país emergente como Colombia, y qué efectos tienen estas variables sobre la intensidad de la innovación.

\section{Conclusiones}

De acuerdo con los resultados encontrados en la presente investigación, las cinco variables asociadas a la financiación que más influyen en la intensificación de las actividades de innovación por parte de las pymes colombianas están asociadas al costo de ajuste, al tamaño de la empresa en número de empleados, a la aversión al riesgo del gerente, al costo esperado de quiebra por innovación y al costo esperado de quiebra en general. Asimismo, a partir de la construcción de gráficos marginales para hallar un efecto aproximado de cada variable sobre la intensidad de la innovación en una empresa "promedio", se encontró que el costo esperado de quiebra por innovación, el tamaño por empleados, la aversión al riesgo del gerente y el costo de ajuste tienen un efecto negativo en la intensificación de la innovación, mientras que el costo esperado de quiebra en general tiene un efecto positivo en la intensificación de las actividades de la innovación en las pymes. 
Como conclusión, se puede sugerir que las variables más importantes para la intensidad de la innovación de las pymes colombianas no se relacionan primariamente con la financiación y sus fuentes. Excepto las variables de control, las variables más definitivas corresponden al costo de ajuste y a variables asociadas al riesgo de innovar y a cómo lo afronta el gerente, incluyendo por ejemplo la aversión al riesgo del gerente y el costo esperado de quiebra general y por innovación. Las características del crédito y las fuentes de financiación al corto plazo son rasgos asociados a la intensidad de la innovación con una presencia solo secundaria en la jerarquía, a pesar de que la literatura se ha enfocado especialmente en temas asociados a las fuentes de financiación y a la estructura de capital. Ciertos tipos de obstáculos para innovar al igual que la asimetría de la información sobre los proyectos de innovación tienen igualmente una importancia secundaria, aunque desde el punto de vista de la economía de la innovación la asimetría de la información haya ocupado gran parte de la atención académica. Curiosamente, entre las variables menos importantes para intensificar la innovación se incluyen algunas como la financiación obtenida para la innovación, las redes de cooperación, el spillover, la educación de los empleados y el perfil de gerente (en términos de su educación y experiencia).

La principal limitación de este estudio, sin embargo, está asociada al uso de un único país arquetípico para la extracción de la muestra. Desafortunadamente, ya que variables tales como la eficiencia de los mercados, la legislación financiera e impositiva o los subsidios son aproximadamente homogéneos en todo el territorio del país estudiado, no fue posible, en términos prácticos, incluir el efecto de estas variables en los modelos (por tener una varianza nula o casi nula).

A pesar de lo anterior, los resultados hallados podrían permitir precisamente que los organismos estatales, las cámaras de comercio y las asociaciones industriales de Colombia reencaucen sus esfuerzos, de ser necesario, para impactar sobre aquellas variables que la evidencia destaca como las de mayor influencia en la intensificación de la innovación. Por ejemplo, la disponibilidad de créditos para aumentar o redirigir la capacidad productiva podría mitigar los costos de ajuste, mientras que el ofrecimiento de garantías parciales adicionales a las empresas que innoven podría reducir los efectos de la aversión al riesgo del gerente y del costo esperado de quiebra por innovación. Una extensión del presente trabajo podría examinar qué tipo de instrumentos de política pública y de fomento a la innovación podrían ser los más adecuados para fomentar la innovación, y de antemano se esperaría que fueran instrumentos que pudieran causar el mayor impacto al menor costo sobre las variables más importantes de acuerdo con el presente estudio.

Finalmente, en cuanto a las implicaciones metodológicas de este trabajo, el mismo introduce un método innovador a la literatura de la innovación, método que podría emplearse en problemas de investigación similares, como lo es, por ejemplo, la búsqueda de los principales determinantes no financieros de la innovación.

\section{Apéndice en línea}

El Apéndice en línea está disponible en https://rebrand.ly/ImpYEfec y describe el proceso de ingeniería de rasgos, las definiciones de las variables y las fuentes en las que se inspiran.

\section{Financiación}

Esta investigación fue financiada por Minciencias (Convocatoria 617 de 2013) y la Universidad del Valle (Convocatoria para estudiantes de doctorado de 2016). 


\section{Referencias}

Aboody, D., \& Lev, B. (2000). Information asymmetry, R\&D, and insider gains. The Journal of Finance, 55(6), 2747-2766. https://doi.org/10.1111/0022-1082.00305

Acharya, V. V., \& Subramanian, K. V. (2009). Bankruptcy codes and innovation. The Review of Financial Studies, 22(12), 4949-4988. https://doi.org/10.1093/rfs/hhp019

Aghion, P., Bloom, N., Blundell, R., Griffith, R., \& Howitt, P. (2002). Competition and innovation: An inverted U relationship. The Quarterly Journal of Economics, 120(2), 701-728. https://doi.org/10.3386/w9269

Álvarez, R., \& Crespi, G.A. (2015). Heterogeneous effects of financial constraints on innovation: Evidence from Chile. Science and Public Policy, 42(5), 711-724. https://doi.org/10.1093/scipol/scu091

Banco de la República de Colombia (2017). Salario mínimo legal de Colombia. Recuperado de https://www.banrep.gov.co/es/estadisticas/salarios\#gsc.tab $=0$

Barona-Zuluaga, B., \& Rivera-Godoy, J.A. (2017). Análisis comparativo de la inversión-financiación de la innovación entre sectores manufacturero y de servicios en Colombia. Libre Empresa, 14(1), 11-27. https://doi.org/10.18041/libemp.2017.v14n1.27101

Barona-Zuluaga, B., Rivera-Godoy, J. A., Aguilera-Cifuentes, C. I., \& Garizado-Román, P. A. (2015). Financiación de la innovación en Colombia. Entramado, 11(1), 80-93. https://doi.org/10.18041/entramado.2015v11n1.21126

Beaudry, C., \& Allaoui, S. (2012). Impact of public and private research funding on scientific production: The case of nanotechnology. Research Policy, 41(9), 1589-1606. https://doi.org/10.1016/j.respol.2012.03.022

Berger, A., \& Udell, G. (1998). The economics of small business finance: The roles of private equity and debt markets in the financial growth cycle. Journal of Banking \& Finance, 22(6-8), 613-673. https://doi.org/10.1016/S0378-4266(98)00038-7

Botta, A. (2017). The complex inequality-innovation-public investment nexus: What we (don't) know, what we should know and what we have to do. Forum for Social Economics, 46(3), 275-298. https://doi.org/10.1080/07360932.2016.1150867

Brancati, E. (2015). Innovation financing and the role of relationship lending for SMEs. Small Business Economics, 44(2), 449-473. https://doi.org/10.1007/s11187-014-9603-3

Brown, J.R., Fazzari, S.M., \& Petersen, B.C. (2009). Financing innovation and growth: Cash flow, external equity, and the 1990s R\&D boom. The Journal of Finance, 64(1), 151-185. https://doi.org/10.1111/j.1540-6261.2008.01431.x

Brown, J.R., Martinsson, G., \& Petersen, B.C. (2012). Do financing constraints matter for R\&D? European Economic Review, 56(8), 1512-1529. https://doi.org/10.1016/j.euroecorev.2012.07.007

Campbell, I. (2007). Chi-squared and Fisher-Irwin tests of two-by-two tables with small sample recommendations. Statistics in Medicine, 26(19), 3661-3675. https://doi.org/10.1002/sim.2832

Canepa, A., \& Stoneman, P. (2007). Financial constraints to innovation in the UK: Evidence from CIS2 and CIS3. Oxford Economic Papers, 60(4), 711-730. https://doi.org/10.1093/oep/gpm044 
Carayannis, E.G., \& Provance, M. (2008). Measuring firm innovativeness: Towards a composite innovation index built on firm innovative posture, propensity and performance attributes. International Journal of Innovation and Regional Development, 1(1), 90-107. https://doi.org/10.1504/IJIRD.2008.016861

Chen, H.-L., Hsu, W.-T., \& Huang, Y.-S. (2010). Top management team characteristics, R\&D investment and capital structure in the IT industry. Small Business Economics, 35(3), 319-333. https://doi.org/10.1007/s11187-008-9166-2

Chien, S.-C., Wang, T.-Y., \& Lin, S.-L. (2010). Application of neuro-fuzzy networks to forecast innovation performance - The example of Taiwanese manufacturing industry. Expert Systems with Applications, 37(2), 1086-1095. https://doi.org/10.1016/j.eswa.2009.06.107

Cucculelli, M. (2018). Firm age and the probability of product innovation. Do CEO tenure and product tenure matter? Journal of Evolutionary Economics, 28(1), 153-179. https://doi.org/https://doi.org/10.1007/s00191-017-0542-4

Czarnitzki, D. (2006). Research and development in small and medium-sized enterprises: The role of financial constraints and public funding. Scottish Journal of Political Economy, 53(3), 335-357. https://doi.org/10.1111/j.1467-9485.2006.00383.x

Czarnitzki, D., \& Hottenrott, H. (2010). Financing constraints for industrial innovation: What do we know? SSRN Electronic Journal. https://doi.org/10.2139/ssrn.1749386

Czarnitzki, D., Hottenrott, H., \& Thorwarth, S. (2011). Industrial research versus development investment: The implications of financial constraints. Cambridge Journal of Economics, 35(3), 527544. https://doi.org/10.2139/ssrn.1494312

Damanpour, F. (1991). Organizational innovation: A meta-analysis of effects of determinants and moderators. Academy of Management Journal, 34(3), 555-590. https://doi.org/10.2307/256406

DANE (2017). Encuesta de desarrollo e innovación tecnológica en la industria manufacturera-EDIT VIII [Conjunto de datos]. https://microdatos.dane.gov.co/index.php/catalog/553/datafile/F37/V10700

DANE (2018). Encuesta de desarrollo e innovación tecnológica en el sector servicios EDITS VI. [Conjunto de datos]. https://microdatos.dane.gov.co/index.php/catalog/584/datafile/F35/V10179

EMIS (2017). Financial statements - Colombian companies (real sector). [Conjunto de datos]. https://www.emis.com

Farlex Financial Dictionary (2012). Adjustment costs. Farlex Financial Dictionary. http://financialdictionary.thefreedictionary.com/Adjustment+Costs

Fernández, V. (2017). The finance of innovation in Latin America. International Review of Financial Analysis, 53, 37-47. https://doi.org/10.1016/j.irfa.2017.08.008

Galia, F., \& Legros, D. (2004). Complementarities between obstacles to innovation: Evidence from France. Research Policy, 33(8), 1185-1199. https://doi.org/10.1016/j.respol.2004.06.004

Galia, F., Mancini, S., \& Morandi, V. (19-21 de junio de 2012). Obstacles to innovation: What hampers innovation in France and Italy [conferencia]. Druid Society Conference 2012 Innovation and Competitiveness. Copenhagen, https://citeseerx.ist.psu.edu/viewdoc/download?doi=10.1.1.477.4363\&rep=rep1\&type=pdf 
García-Pérez de Lema, D., Barona-Zuluaga, B., \& Madrid-Guijarro, A. (2013). Financiación de la innovación en las mipyme iberoamericanas. Estudios Gerenciales, 29(126), 12-16. https://doi.org/10.1016/S0123-5923(13)70015-9

García-Quevedo, J., Segarra-Blasco, A., \& Teruel, M. (2018). Financial constraints and the failure of innovation projects. Technological Forecasting and Social Change, 127, 127-140. https://doi.org/10.1016/j.techfore.2017.05.029

Ghadim, A.K.A., Pannell, D.J., \& Burton, M.P. (2005). Risk, uncertainty, and learning in adoption of a crop innovation. Agricultural Economics, 33(1), 1-9. https://doi.org/10.1111/j.15740862.2005.00433.x

Ghisetti, C., \& Montresor, S. (2020). On the adoption of circular economy practices by small and medium-size enterprises (SMEs): Does "financing-as-usual" still matter? Journal of Evolutionary Economics, 30(2), 559-586. https://doi.org/10.1007/s00191-019-00651-w

Guariglia, A., \& Liu, P. (2014). To what extent do financing constraints affect Chinese firms' innovation activities? International Review of Financial Analysis, 36(C), 223-240. https://doi.org/10.1016/j.irfa.2014.01.005

Hahn, D., Minola, T., Vismara, S., \& De Stasio, V. (2019). Financing innovation: Challenges, opportunities, and trends. Foundations and Trends ${ }^{\circledR}$ in Entrepreneurship, 15(3-4), 328-367. https://doi.org/10.1561/0300000085-1

Hall, B.H. (2010). The financing of innovative firms. Review of Economics and Institutions, 14(2), 829. https://doi.org/10.5202/rei.v1i1.4

Hall, B. H., \& Lerner, J. (2010). The financing of R\&D and innovation. En B. H. Hall \& N. Rosenberg (Eds.), Handbook of the economics of innovation ( $1^{\text {a }}$ ed., Vol. 1, pp. 609-639). Amsterdam: Elsevier North Holland. https://doi.org/10.1016/S0169-7218(10)01014-2

Hall, B. H., \& Rosenberg, N. (2010). Introduction of the handbook. En B. H. Hall \& N. Rosenberg (Eds.), Handbook of the economics of innovation ( $1^{\text {a }}$ ed., Vol. 1, pp. 3-9). Amsterdam: Elsevier North Holland. https://doi.org/10.1016/S0169-7218(10)01001-4

Higgins, R. C. (2012). Analysis for financial management (10a ed.). New York, NY: McGrawHill/Irwin.

Himmelberg, C. P., \& Petersen, B. C. (1994). R\&D and internal finance: A panel study of small firms in high-tech industries. The Review of Economics and Statistics, 76(1), 38-51. https://doi.org/10.2307/2109824

Ho, R. (2012). Big data machine learning: Patterns for predictive analytics . DZone Refcardz, Vol. 2014. http://refcardz.dzone.com/refcardz/machine-learning-predictive

Holmstrom, B. (1989). Agency costs and innovation. Journal of Economic Behavior \& Organization, 12(3), 305-327. https://doi.org/10.1016/0167-2681(89)90025-5

Hottenrott, H., \& Richstein, R. (2020). Start-up subsidies: Does the policy instrument matter? Research Policy, 49(1), 103888. https://doi.org/10.1016/j.respol.2019.103888

Howell, S.T. (2017). Financing innovation: Evidence from R\&D grants. American Economic Review, 107(4), 1136-1164. https://doi.org/10.1257/aer.20150808

Hsu, P.-H., Tian, X., \& Xu, Y. (2014). Financial development and innovation: Cross-country evidence. 
Journal of Financial Economics, 112(1), 116-135. https://doi.org/10.1016/j.jfineco.2013.12.002

Jensen, M.C., \& Meckling, W.H. (1976). Theory of the firm: Managerial behavior, agency costs and ownership structure. Journal of Financial Economics, 3(4), 305-360. https://doi.org/10.1016/0304405X(76)90026-X

Kingma, D.P., \& Ba, J. (2017). Adam: A method for stochastic optimization. ArXiv. https://arxiv.org/abs/1412.6980.

Kleinknecht, A. (2016). Innovation patterns in crisis and prosperity: Schumpeter's long cycle reconsidered. Berlin: Alemania: Springer.

Kuhn, M., \& Johnson, K. (2013). Applied predictive modeling. Nueva York, NY: Springer.

Kvalseth, T.O. (1985). Cautionary Note about R ${ }^{2}$. The American Statistician, 39(4), 279-285. https://doi.org/10.2307/2683704

Landry, R., Amara, N., \& Lamari, M. (2002). Does Social Capital Determine Innovation? To What Extent? Technological Forecasting and Social Change, 69(7), 681-701. https://doi.org/10.1016/S0040-1625(01)00170-6

Langebaek, A., \& Vásquez, D. (2007). Determinantes de la actividad innovadora en la industria manufacturera colombiana. Borradores de Economía, 433(Marzo), 1-35. https://www.banrep.gov.co/docum/ftp/borra433.pdf

Leiponen, A.E., \& Poczter, S. (2014). Financing of innovation strategies in emerging economies. SSRN Electronic Journal. https://doi.org/10.2139/ssrn.2403494

Ley 905/2004. Por medio de la cual se modifica la Ley 590 de 2000 sobre promoción del desarrollo de las micro, pequeña y mediana empresa Colombiana y se dictan otras disposiciones. Diario Oficial No. 45.628 de 2 de agosto de 2004, Colombia: Congreso de la República de Colombia. http://www.secretariasenado.gov.co/senado/basedoc/ley_0905_2004.html

Lintner, J. (1965). The valuation of risk assets and the selection of risky investments in stock portfolios and capital budgets. The Review of Economics and Statistics, 47(1), 13-37. https://doi.org/10.2307/1924119

Lussuamo, J.M., \& Serrasqueiro, Z. (2020). Restrictions on access to bank finance for SMEs in Cabinda-Angola. Small Enterprise Research, 27(3), 275-288. https://doi.org/10.1080/13215906.2020.1835705

Mancusi, M.L., \& Vezzulli, A. (2014). R\&D and credit rationing in SMEs. Economic Inquiry, 52(3), 1153-1172. https://doi.org/10.1111/ecin.12080

Markman, G.D., Balkin, D.B., \& Schjoedt, L. (2001). Governing the innovation process in entrepreneurial firms. The Journal of High Technology Management Research, 12(2), 273-293. https://doi.org/10.1016/S1047-8310(01)00040-2

Mazzucato, M. (2013). Financing innovation: Creative destruction vs. destructive creation. Industrial and Corporate Change, 22(4), 851-867. https://doi.org/10.1093/icc/dtt025

Menshchikova, V.I., \& Sayapin, A.V. (2016). Model of innovation-oriented state economic policy. European Research Studies Journal, XIX(1), 189-200. https://doi.org/10.35808/ersj/514

Merz, M. (2019). Innovative efficiency as a lever to overcome financial constraints in R\&D contests. 
Economics of Innovation and New Technology, 30(3), 284-294. https://doi.org/10.1080/10438599.2019.1695946

Modigliani, F., \& Miller, M.H. (1958). The cost of capital, corporation finance and the theory of investment. The American Economic Review, 48(3), 261-297. http://www.jstor.org/stable/1809766

Mohnen, P., Palm, F.C., van der Loeff, S.S., \& Tiwari, A. (2008). Financial constraints and other obstacles: Are they a threat to innovation activity? De Economist, 156(2), 201-214. https://doi.org/10.1007/s10645-008-9089-y

Molnar, C. (2020). Interpretable machine learning. A guide for making black box models explainable. https://christophm.github.io/interpretable-ml-book/

Moon, B. (2021). Strategic R\&D projects choice of the firm: Theory and evidence from Korea. Technology Analysis \& Strategic Management, 33(5), 465-475. https://doi.org/10.1080/09537325.2019.1644310

Mossin, J. (1966). Equilibrium in a capital asset market. Econometrica, 34(4), 768-783. https://doi.org/10.2307/1910098

Myers, S.C. (1984). Capital structure puzzle. The Journal of Finance, 39(3), 574-592. https://doi.org/10.3386/w1393

Nor, N.G.M., Bhuiyan, A.B., Said, J., \& Alam, S.S. (2016). Innovation barriers and risks for food processing SMEs in Malaysia: A logistic regression analysis. Malaysian Journal of Society and Space, 12(2), 167 - 178.

O'Brien, J.P. (2003). The capital structure implications of pursuing a strategy of innovation. Strategic Management Journal, 24(5), 415-431. https://doi.org/10.1002/smj.308

OECD/Eurostat (2018). Oslo Manual 2018: Guidelines for Collecting, Reporting and Using Data on Innovation, 4th Edition. The Measurement of Scientific, Technological and Innovation Activities. OECD Publishing, Paris/Eurostat, Luxemburgo. https://doi.org/10.1787/9789264304604-en

OECD/Eurostat (2005). Oslo Manual: Guidelines for Collecting and Interpreting Innovation Data, 3rd Edition. The Measurement of Scientific and Technological Activities. OECD Publishing, Paris, https://doi.org/10.1787/9789264013100-en

Padilla-Ospina, A.M., Medina-Vásquez, J.E., \& Ospina-Holguín, J.H. (2021). Financial determinants of innovation in SMEs: A machine learning approach. Journal of Small Business Strategy, 31(5), 117-131. https://doi.org/10.53703/001c.29839

Padilla-Ospina, A.M., Medina-Vásquez, J.E., \& Rivera-Godoy, J.A. (2018). Financing innovation: A bibliometric analysis of the field. Journal of Business \& Finance Librarianship, 23(1), 63-102. https://doi.org/10.1080/08963568.2018.1448678

Padilla-Ospina, A.M., Rivera-Godoy, J.A., \& Ospina-Holguín, J.H. (2015). Determinantes de la estructura de capital de las mypimes del sector real participantes del Premio Innova. Revista $\begin{array}{lllll}\text { Finanzas } & y & \text { Politica } & \text { Económica, } & \text { 15(1), }\end{array}$ https://doi.org/10.14718/revfinanzpolitecon.2015.7.2.8

Rivera-Godoy, J.A. (2015). Análisis de la relación de la innovación empresarial con la financiación en Colombia. Cuadernos de Administración, 28(50), 11-37. https://doi.org/10.11144/Javeriana.cao2850.arie 
Romijn, H., \& Albaladejo, M. (2002). Determinants of innovation capability in small electronics and software firms in Southeast England. Research Policy, 31(7), 1053-1067. https://doi.org/10.1016/S0048-7333(01)00176-7

Ross, S.A. (1976). The arbitrage theory of capital asset pricing. Journal of Economic Theory, 13(3), 341-360. https://doi.org/10.1016/0022-0531(76)90046-6

Serrasqueiro, Z., Nunes, P.M., \& da Rocha Armada, M. (2016). Capital structure decisions: Old issues, new insights from high-tech small- and medium-sized enterprises. The European Journal of Finance, 22(1), 59-79. https://doi.org/10.1080/1351847X.2014.946068

Sharpe, W.F. (1964). Capital asset prices: A theory of market equilibrium under conditions of risk. The Journal of Finance, 19(3), 425-442. https://doi.org/10.1111/j.1540-6261.1964.tb02865.x

Sierra, J., Malaver, F., Vargas, M., Robledo, J., \& Malaver, F. (2009). La financiación de la innovación: un análisis a partir de la encuesta de innovación de Bogotá y Cundinamarca. En J. R. Velásquez, F. Malaver-Rodríguez, \& M. Vargas-Pérez (Eds.), Encuestas, datos y descubrimiento de conocimiento sobre la innovación en Colombia (pp. 175-210). Bogotá: Colombia: Universidad Nacional de Colombia, Pontificia Universidad Javeriana, Universidad Pontificia Bolivariana y Observatorio Colombiano de Ciencia y Tecnología.

Titterington, M. (2010). Neural networks. Wiley Interdisciplinary Reviews: Computational Statistics, 2(1), 1-8. https://doi.org/10.1002/wics.50

Treynor, J.L. (1961). Market value, time, and risk. SSRN Electronic Journal. https://doi.org/10.2139/ssrn.2600356

Tsur, Y., Sternberg, M., \& Hochman, E. (1990). Dynamic modelling of innovation process adoption with risk aversion and learning. Oxford Economic Papers, 42(2), 336-355. https://doi.org/https://doi.org/10.1093/oxfordjournals.oep.a041950

Wang, T.-Y., \& Chien, S.-C. (2006). Forecasting innovation performance via neural networks-a case of Taiwanese manufacturing industry. Technovation, 26(5-6), 635-643. https://doi.org/10.1016/j.technovation.2004.11.001

Wang, Y., Li, J., \& Furman, J.L. (2017). Firm performance and state innovation funding: Evidence from China's innofund program. Research Policy, 46(6), 1142-1161. https://doi.org/10.1016/j.respol.2017.05.001

$\mathrm{Xu}$, Z. (2020). Economic policy uncertainty, cost of capital, and corporate innovation. Journal of Banking \& Finance, 111, 105698. https://doi.org/10.1016/j.jbankfin.2019.105698

Zhang, D., \& Zheng, W. (2020). Does financial constraint impede the innovative investment? Micro evidence from China. Emerging Markets Finance and Trade, 56(7), 1423-1446. https://doi.org/10.1080/1540496X.2018.1542594

Zhu, Y., Wittmann, X., \& Peng, M. W. (2012). Institution-based barriers to innovation in SMEs in China. Asia Pacific Journal of Management, 29(4), 1131-1142. https://doi.org/10.1007/s10490-0119263-7 